\title{
Primary central nervous system lymphoma
}

Primary central nervous system lymphoma (PCNSL) is an aggressive type of lymphoma that arises in the brain parenchyma or spinal cord, eyes, cranial nerves, and meninges. PCNSL can occur in immunocompetent as well as in immunocompromised hosts, including human immunodeficiency virus (HIV)-infected people or posttransplant patients. PCNSL represented a major cause of morbidity and mortality among HIV-infected people before the introduction of highly active antiretroviral therapy. However, PCNSL nearly disappeared in the era of effective combined antiretroviral therapy ${ }^{[1]}$ Curiously, the incidence of PCNSL has been raising in HIV-uninfected people, ${ }^{[2]}$ in whom PCNSL today represent $<1 \%$ of all non-Hodgkin lymphomas (NHL) and approximately 2-3\% of all brain tumors ${ }^{[3]}$ Irrespective of HIV status, $90-95 \%$ of PCNLS are histologically classified as diffuse large B-cell lymphoma (DLBCL) which consistently express B-cell antigens and monotypic surface immunoglobulin light chains, and usually display positivity for MUM1 (90\% of cases) and BCL6 (60-80\% of cases), with frequent coexpression of BCL2 and MYC. Only a minority $(<10 \%)$ of cases express CD10.. ${ }^{[3]}$ Overall, PCNSL does not homogeneously conform to either of the principal molecular profiles identified in systemic DLBCL, namely germinal center and activated B-cell subtypes, but appears to exhibit unique transcriptional features by gene expression profiling. ${ }^{[4]}$ From a virological point of view PCNSL DLBCL harbor Epstein-Barr virus (EBV) infection only in immunocompromised individuals. ${ }^{[1,3]}$

In their study Mahadevan, et al. ${ }^{[5]}$ analyzed the pathological features of 24 South Indian patients with PCNSL displaying DLBCL histotype. These cases consistently showed centroblastic morphology and uniformly exhibited activated B-cell phenotype with expression of MUM1, but not CD138. They also showed coexpression of BCL6 and MUM1 in 50\% of cases. All cases were negative for EBV by EBER in situ hybridization and latent

\begin{tabular}{|l|l|}
\hline \multicolumn{2}{|c|}{ Access this article online } \\
\hline Quick Response Code: & Website: \\
\hline & www.ruralneuropractice.com \\
\cline { 2 - 3 } & \\
\hline & \\
&
\end{tabular}

membrane protein 1 (LMP1) immunohistochemistry. Overall, these PCNSL arising in Indian patients showed pathologic and molecular features similar to those displayed by PCNS DLBCL arising in other Asian patients, but partly different from those displayed by systemic DLBCL. As discussed by Mahadevan, et al., ${ }^{[5]}$ systemic DLBCL from Asian patients have been shown to have a predominance of GCB origin in some studies and activated B cell origin in other studies.

Taken together these results increase our knowledge on PCNSL of the immunocompetent host. According to this study, PCNSL is a unique clinicopathological entity among DLBCL; it has centroblastic morphology and an origin from late germinal center/early post germinal center exit B cells. These cells, which have not acquired plasmacytic differentiation, show frequent coexpression of MUM 1 and BCL6, but not CD138. Therefore, PCNS DLBCLs differ from systemic DLBCLs because they cannot be subdivided into prognostic groups according to the cell of origin. Moreover, the prognostic significance of molecular alterations identified so far (reviewed $\mathrm{in}^{[6,7]}$ ) remains to be defined.

Besides the cell of origin of PCNS DLBCL the article by Mahadevan, et al., ${ }^{[5]}$ focuses the reader's attention on interesting, but still poorly known, key points such as the cell derivation and pathogenesis of PCNS DLBCL. ${ }^{[8]}$ Because the central nervous system lacks lymph nodes and lymphatics, it has been hypothesized that PCNSL may originate from B cells derived from systemic lymphoid tissues and normally trafficking in and out of the CNS. The precise cellular derivation of PCNSL is however still unknown. The molecular pathogenesis of PCNSL is also poorly understood: existing evidence is mainly restricted to the role of EBV in AIDS-related PCNSL. EBV-infected lymphoma cells frequently express the EBV-encoded oncoprotein LMP1, which, in turn, upregulates the expression of BCL2 ${ }^{[8]}$ It is conceivable that EBV may act as an oncogenic agent or at least as an oncogenic cofactor.

By contrast, EBV has no role in lymphomagenesis of PCNSL in immunocompetent host. On the genetic level PCNS DLBCL of immunocompetent host show ongoing aberrant somatic hypermutation that, besides the IG locus, targets the PAX5, TTF, MYC, and PIM1 genes. Several 
important pathways, including the B-cell receptor (BCR), the toll-like receptor, and the nuclear factor- $\mathrm{KB}$ pathway, are activated. Genomic aberrations in PCNSL include losses on chromosome 6p21 containing human leukocyte antigen (HLA) loci. The basis for specific tropism and localization of lymphoma within the brain are problems crucial to the pathogenesis of PCNSL. Expression of chemokine (C-X-C motif) ligand (CXCL) 12 and CXCL13 within PCNSL has been demonstrated and chemotactic responsiveness to these peptides by CNS lymphoma recently documented, favoring their role as neurotropic factors. Nevertheless, many of these alterations are also present in subgroups of systemic DLBLC and might not be the only reasons for the specific tropism of PCNSL. ${ }^{[6]}$ However, the reported alterations (reviewed in ${ }^{[6,7]}$ ) induce and maintain a proliferative, non-apoptotic phenotype of the B cells, and may in part explain the poor prognosis of patients with PCNSL.

\section{Annunziata Gloghini, Antonino Carbone ${ }^{1}$ \\ Department of Diagnostic Pathology and Laboratory Medicine, Fondazione IRCCS, Milan National Cancer Institute 'IRCCS' Foundation, 'Department of Pathology, CRO Aviano National Cancer Institute, Italy \\ Address for correspondence: Dr. Annunziata Gloghini, Department of Diagnostic Pathology and Laboratory Medicine, Fondazione IRCCS, Istituto Nazionale Tumori, Via G. Venezian 1, Milano I-20133, Italy. E-mail: annunziata.gloghini@istitutotumori.mi.it}

\section{References}

1. Carbone A, Vaccher E, Gloghini A, Pantanowitz L, Abayomi A, de Paoli P, et al. Diagnosis and management of lymphomas and other cancers in HIV-infected patients. Nat Rev Clin Oncol 2014;11:223-38.

2. Olson JE, Janney CA, Rao RD, Cerhan JR, Kurtin PJ, Schiff D, et al. The continuing increase in the incidence of primary central nervous system non-Hodgkin lymphoma: A surveillance, epidemiology, and end results analysis. Cancer 2002;95:1504-10.

3. Kluin PM, Deckert M, Ferry JA. Primary diffuse large B-cell lymphoma of the CNS. In: Swerdlow SH, Campo E, Harris NL, Jaffe ES, Pileri SA, Stein H, et al., editors. WHO Classification of Tumours of Haematopietic and Lymphoid Tissues. $4^{\text {th }}$ ed. Lyon, France: IARC Press; 2008. p. 240-1.

4. Tun HW, Personett D, Baskerville KA, Menke DM, Jaeckle KA, Kreinest $\mathrm{P}$, et al. Pathway analysis of primary central nervous system lymphoma. Blood 2008;111:3200-10.

5. Mahadevan A, Rama Rao C, Shanmugham M, Shankar SK. Primary central nervous system diffuse large B-cell lymphoma in the immunocompetent: Immunophenotypic subtypes and EBV association. J Neurosc Rural Pract 2015;6:10-6.

6. Deckert M, Montesinos-Rongen M, Brunn A, Siebert R. Systems biology of primary CNS lymphoma: From genetic aberrations to modeling in mice. Acta Neuropathol 2014;127:175-88.

7. Wang CC, Carnevale J, Rubenstein JL. Progress in central nervous system lymphomas. Br J Haematol 2014;166:311-25.

8. Larocca LM, Capello D, Rinelli A, Nori S, Antinori A, Gloghini A, et al. The molecular and phenotypic profile of primary central nervous system lymphoma identifies distinct categories of the disease and is consistent with histogenetic derivation from germinal center-related B cells. Blood 1998;92:1011-9.

How to cite this article: Gloghini A, Carbone A. Primary central nervous system lymphoma. J Neurosci Rural Pract 2015;6:2-3.

Source of Support: Nil. Conflict of Interest: None declared.

\section{"Quick Response Code" link for full text articles}

The journal issue has a unique new feature for reaching to the journal's website without typing a single letter. Each article on its first page has a "Quick Response Code". Using any mobile or other hand-held device with camera and GPRS/other internet source, one can reach to the full text of that particular article on the journal's website. Start a QR-code reading software (see list of free applications from http://tinyurl.com/ yzlh2tc) and point the camera to the QR-code printed in the journal. It will automatically take you to the HTML full text of that article. One can also use a desktop or laptop with web camera for similar functionality. See http://tinyurl.com/2bw7fn3 or http://tinyurl.com/3ysr3me for the free applications. 